\title{
INVESTIGATING 2MASS J06593158-0405277: AN FUor BURST IN A TRIPLE SYSTEM?
}

\author{
A. Caratti o Garatti ${ }^{1}$, R. Garcia Lopez ${ }^{1}$, T. P. Ray ${ }^{1}$, J. Eislöffel ${ }^{2}$, B. Stecklum ${ }^{2}$, \\ A. Scholz $^{3}$, S. Kraus ${ }^{4}$, G. Weigelt ${ }^{5}$, A. Kreplin ${ }^{4}$, and V. Shenavrin ${ }^{6}$ \\ ${ }^{1}$ School of Cosmoc Physics, Dublin Institute for Advanced Studies, 31 Fitzwilliam Place, Dublin 2, Ireland; alessio@cp.dias.ie \\ ${ }_{2}$ Thüringer Landerssternwarte Tautenburg, Sternwarte 5, Tautenburg, Germany \\ ${ }^{3}$ School of Physics and Astronomy, University of St Andrews, North Haugh, St Andrews KY16 9SS, UK \\ ${ }^{4}$ School of Physics, University of Exeter, Physics Building, Stocker Road, Exeter EX4 4QL, UK \\ ${ }^{5}$ Max Planck Institut für Radioastronomie, Auf dem Hügel 69, D-53121 Bonn, Germany \\ ${ }^{6}$ Lomonosov Moscow State University, Sternberg Astron. Inst., Universitetsky pr. 13, 119234 Moscow, Russia \\ Received 2015 May 5; accepted 2015 May 12; published 2015 June 2
}

\begin{abstract}
FUor outbursts in young stellar objects are the most dramatic events among episodic accretion phenomena. The origin of these bursts is not clear: disk instabilities and/or disk perturbations by an external body being the most viable hypotheses. Here, we report our Very Large Telescope/SINFONI high angular resolution AO-assisted observations of 2MASS J06593158-0405277, which is undergoing a recently discovered FUor outburst. Our observations reveal the presence of an extended disk-like structure around the FUor, a very low-mass companion (2MASS J06593158-0405277B) at 100 AU in projection, and, possibly, a third closer companion at $\sim 11$ AU. These sources appear to be young, displaying accretion signatures. Assuming the components are physically linked, 2MASS J06593158-0405277 would then be one of the very few triple systems observed in FUors.
\end{abstract}

Key words: binaries: close - circumstellar matter - stars: formation - stars: individual (2MASS J065931580405277) - stars: pre-main sequence - techniques: high angular resolution

\section{INTRODUCTION}

FU Orionis-like outbursts (hereafter FUors) are the most dramatic events among episodic accretion phenomena in young stellar objects (YSOs), displaying a mass accretion rate $\left(\dot{M}_{\text {acc }}\right)$ increase by several orders of magnitude (up to $\dot{M}_{\text {acc }} \sim 10^{-4} M_{\odot}$ $\mathrm{yr}^{-1}$; see, e.g., Hartmann \& Kenyon 1996; Audard et al. 2014). As a result, the YSO brightness is boosted by 5 mag or more in the optical (e.g., Herbig 1977). These bursts have a dramatic impact on the YSO circumstellar environment (Baraffe et al. 2012; Vorobyov et al. 2013; Audard et al. 2014). The rise times of these outbursts are usually short (from several months to a few years), whereas the decay timescales range from years to several decades. Spectra of FUor progenitors have only been obtained in two cases, both indicating that they are low-mass YSOs (e.g., Herbig 1977; Miller et al. 2011).

The origin of bursts in FUors is not well understood, and disk instabilities, disk fragmentation, and/or disk perturbation by an external body are the most viable hypotheses (see review by Audard et al. 2014 and references therein). Several models with thermal or gravitational plus magneto-rotational instabilities are able to mimic the main characteristics of the outbursts (see, e.g., Clarke et al. 2005; Zhu et al. 2010a, 2010b). Notably, the recent version of the disk gravitational instability and fragmentation model by Vorobyov \& Basu (2015) reproduces the physical properties including the episodic nature of the bursts. Aside from internal instabilities, external triggers, namely close encounters in binary or multiple systems, can produce FUor bursts (Bonnell \& Bastien 1992; Reipurth \& Aspin 2004; Reipurth et al. 2014).

FUors are rare transient phenomena, and they have usually been detected and studied in detail several months or even years after the beginning of the outburst. 2MASS J065931580405277 is a YSO undergoing an FU Ori-type outburst (Hillenbrand 2014; Maehara et al. 2014). The outburst was detected on the 2014 November 23 (Maehara et al. 2014), and previous observations indicate that the object began its slow rise at the end of 2013 and reached its maximum at the end of 2014 (Hackstein et al. 2014; Kóspál et al. 2015). From archival photometric data, Kóspál et al. (2015) identify the progenitor as a low-mass T Tauri star $\left(0.75 M_{\odot}\right)$ with a circumstellar disk of $0.01-0.06 M_{\odot}$, and an age of $\sim 6 \times 10^{5}$ years.

\section{OBSERVATIONS AND DATA REDUCTION}

2MASS J06593158-0405277 was observed with ESO/Very Large Telescope UT4 on 2015 January 25 with the nearinfrared (near-IR) integral field spectrograph SINFONI (Eisenhauer et al. 2003) in the $J(1.1-1.4 \mu \mathrm{m})$ and $K(1.95-2.45 \mu \mathrm{m})$ bands with a spectral resolution of $R \sim 2000$ and 4000, respectively. The observations were performed with the highest spatial sampling (12.5 mas pixel scale, and field of view-FoV -of $0 . \prime 8 \times 0 . \prime 8$ ) and in AO-assisted mode (using the target as a natural guide star) with a mean optical seeing of $\sim 0$ ". 8 , which provided a FWHM (measured on the point-spread functionPSF) of $\sim 50$ and $\sim 70$ mas in the $J$ and $K$ band, respectively. The detector integration time (DIT), number of sub-exposures per frame (NDITS) and exposures were $30 \mathrm{~s}, 4$, and 5 ( $J$ band), and $15 \mathrm{~s}, 5$, and 7 ( $K$ band), for a total integration time onsource of 600 and $525 \mathrm{~s}$ in the $J$ and $K$ band, respectively. An additional photometric standard star (HD 42655) was observed for flux calibration of the data cubes and removal of telluric features from the spectra. Data were reduced using the SINFONI data reduction pipeline in GASGANO (Modigliani et al. 2007), that is, dark and bad pixel masks, flat-field corrections, optical distortion correction, and wavelength calibration with arc lamps.

Two spectra were extracted from each data cube using a $5 \times 5$ pixel area centered at the position of the peak flux of the FUor and its companion ( $B$, see Section 3). Due to the FUor brightness and its spatially extended disk-like emission (see 

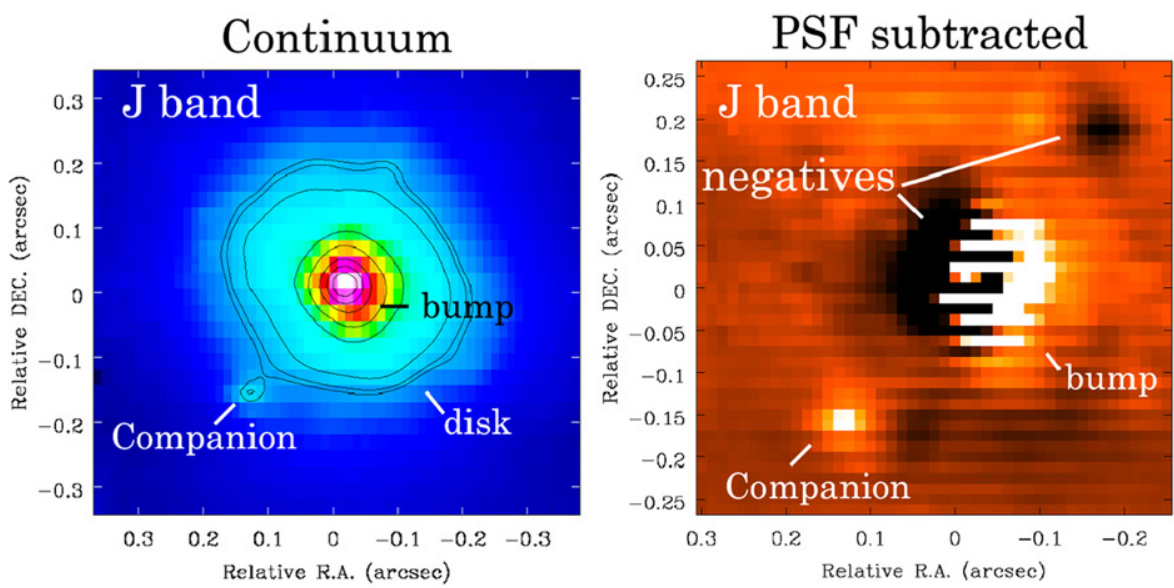

\section{Continuum subtracted}
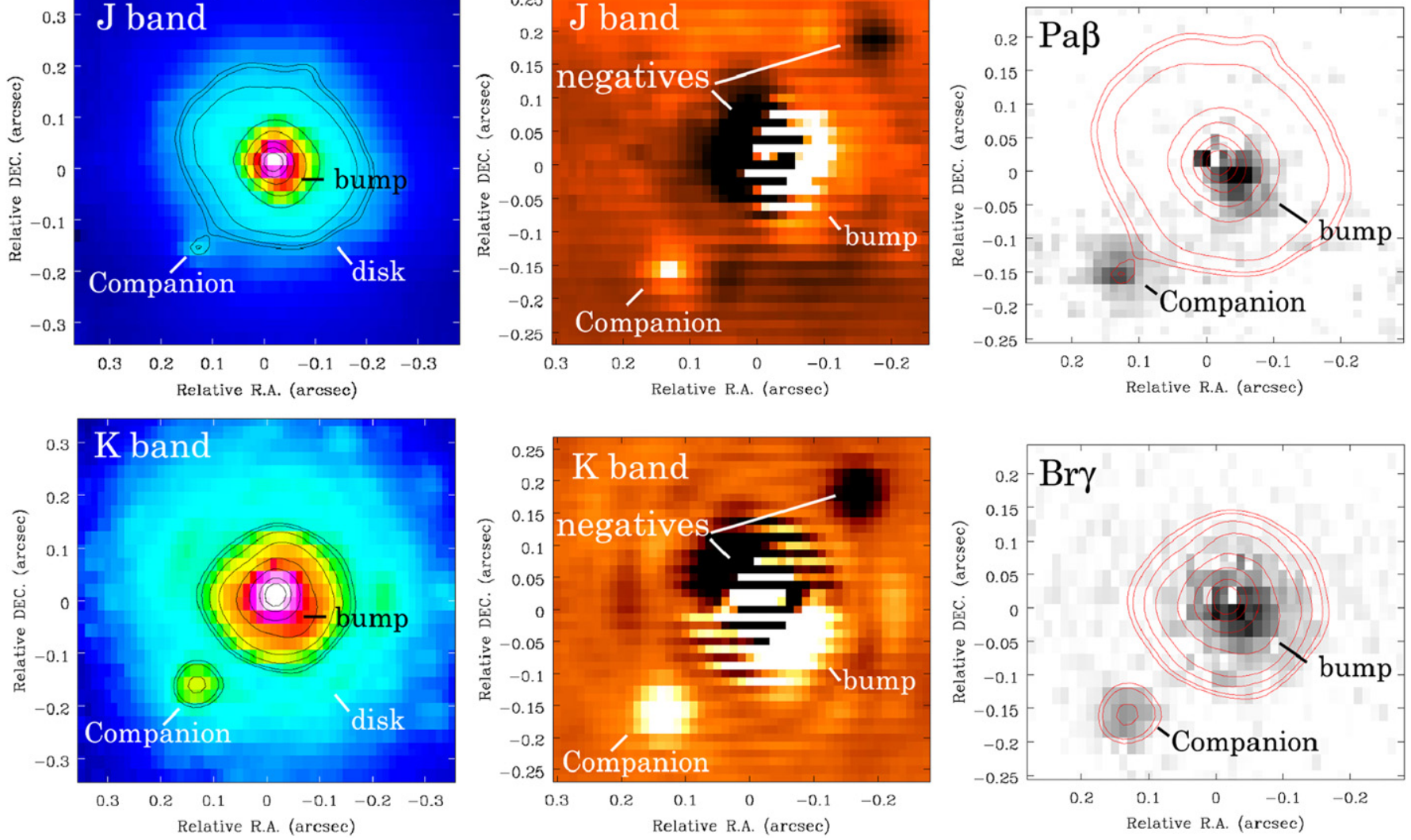

Figure 1. Left: SINFONI $J$ - (upper panel) and $K$-band (lower panel) images of 2MASS J06593158-0405277 showing the disk-like feature, the "bump" position, and the companion $(B)$. Center: difference $J$ - (upper panel) and $K$-band (lower panel) images showing the "bump." The PSF was removed by subtracting the same image rotated by $180^{\circ}$. Right: $\mathrm{Pa} \beta$ (upper panel) and $\mathrm{Br} \gamma$ (lower panel) continuum-subtracted grayscale images. The same continuum contours are shown as in the left panels.

Section 3), a background spectrum was extracted close to the companion position and subtracted from its spectrum to remove the contamination from the FUor source.

Additionally, continuum images (at 1.25 and $2.2 \mu \mathrm{m}$ ) and continuum-subtracted $\mathrm{Pa} \beta$ and $\mathrm{Br} \gamma$ images were created following the same procedures as in Beck et al. (2008) and Garcia Lopez et al. (2013).

As no PSF standard was observed, to remove the FUor's PSF from the continuum and evaluate the asymmetry of the bright central core (see Section 3), we rotate the continuum images by $180^{\circ}$, match the PSF and subtract the rotated images from the original ones in both $J$ and $K$ bands.

\section{RESULTS}

As illustrated in Figure 1, SINFONI data cubes in both bands show three main features around 2MASS J06593158-0405277: (i) a faint companion (hereafter 2MASS J06593158$0405277 \mathrm{~B}$ or $B$ ) located at 227 mas (or $102 \mathrm{AU}$ in projection at a distance of $450 \mathrm{pc}^{7}$; see Kóspál et al. 2015), southeast from the central source (see left panels of Figure 1); (ii) an extended and elliptical disk-like structure around the FUor (size $\sim 0$ ". 2 or $90 \mathrm{AU}$ at a distance of $450 \mathrm{pc}$ ), possibly scattered light from the disk or circumstellar nebulosity (e.g., Goodrich 1987; see also left panels of Figure 1); (iii) a bright asymmetric central

\footnotetext{
The distance to the object is highly uncertain as well as its association with the molecular cloud L 1650, $d \sim 2.3 \mathrm{Kpc}$ (Kim et al. 2004).
}

core of the FUor source, which has a "bump" toward the southwest, clearly detected after subtracting the FUor's rotated images (see central panels of Figure 1). Moreover, both $\mathrm{Pa} \beta$ and $\mathrm{Br} \gamma$ lines in emission are detected at the position of 2MASS J06593158-0405277 B and near the continuum emission of the "bump," as shown in the right panels of Figure 1.

\subsection{The Companion: 2MASS J06593158-0405277B}

On the basis of Gaussian fitting of both $J$ - and $K$-band data cubes, we infer the following coordinates for $B$ : R.A. $(\mathbf{J} 2000)=06: 59: 31.599$, decl. $(\mathbf{J} 2000)=-04: 05: 28.01$, that is, its separation from the FUor is $\Delta \alpha=0$ "' $15, \Delta \delta=-0$ ". 17 , with a P.A. $=131: 4$. Our PSF fitting allows us to derive $J$ - and $K$-band magnitudes of $14.8 \pm 0.4$ and $12.9 \pm 0.2 \mathrm{mag}$, respectively. The photometric uncertainties mostly arise from the bright background due to the scattered light from the FUor. In the upper panel of Figure 2, we show the flux calibrated spectrum in the $J$ and $K$ bands. The spectrum was obtained after subtracting the FUor scattered light in the background (see Section 2). The spectrum shows several broad molecular absorption bands from $\mathrm{H}_{2} \mathrm{O}(1.1,1.4,1.9$, and $2.4 \mu \mathrm{m}), \mathrm{CO}$ (bandheads $v=2-0,3-1,4-2,5-3$ from $2.29 \mu \mathrm{m}$ in the $K$ band), and, possibly, $\mathrm{FeH}$ in the $J$ band, as well as atomic absorption lines ( $\mathrm{Ca}$ I and $\mathrm{Na}$ I) in the $K$ band. These absorption features have a photospheric origin, and are distinctive of late M-type stars (see, e.g., Cushing et al. 2005; Rayner et al. 2009; Scholz et al. 2009). We use near-IR spectra of very low-mass 


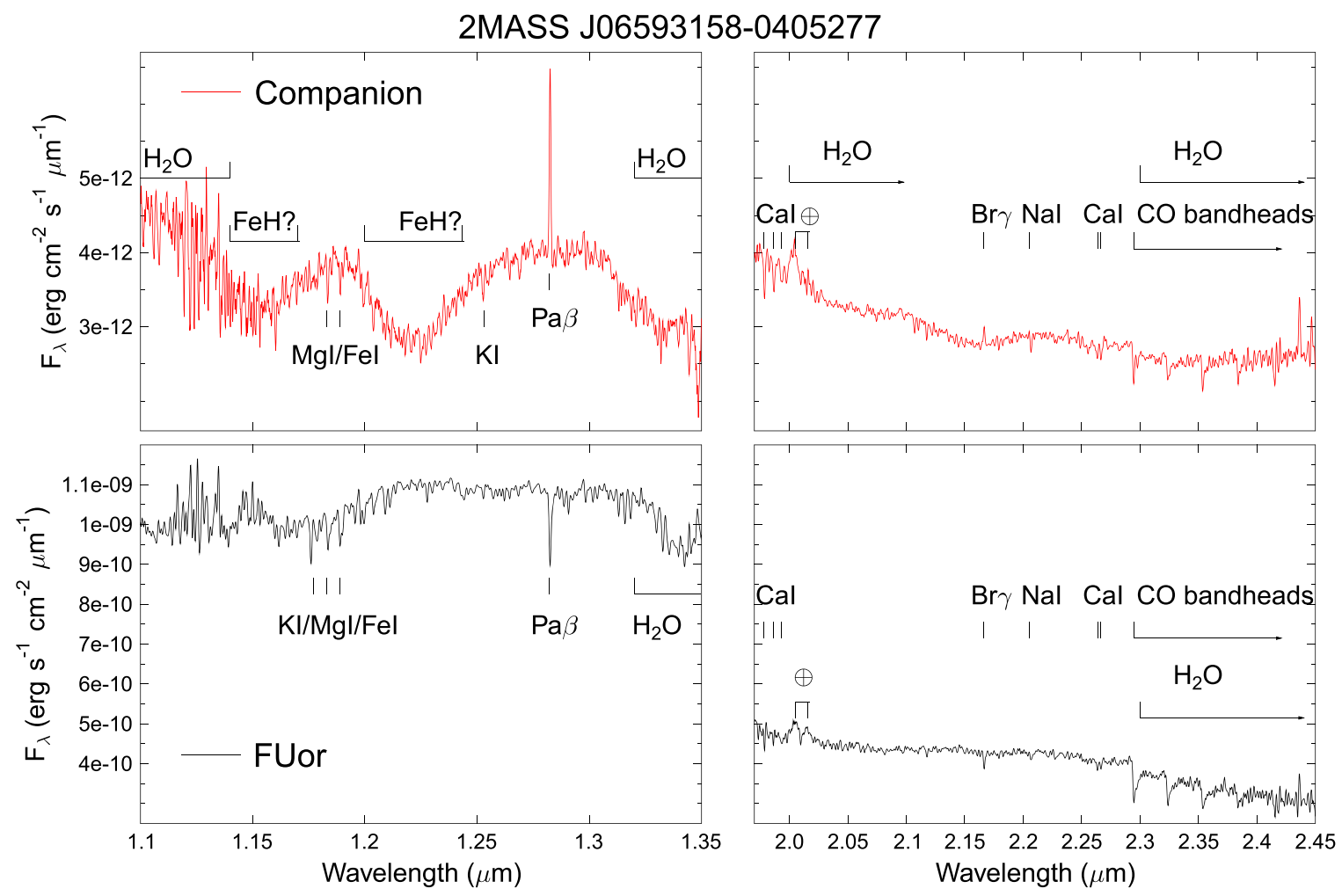

Figure 2. SINFONI $J$ - and $K$-band flux calibrated spectra of 2MASS J06593158-0405277B (red solid line; upper panels) and of the FUor (black solid line; lower panels). The most prominent features are also labeled.

and brown-dwarfs YSOs from Scholz et al. (2012) to constrain the spectral type $(\mathrm{SpT})$ of $B$. The inferred $\mathrm{SpT}$ is between M5 and M9, that is $\mathrm{SpT}=\mathrm{M} 7 \pm 2$, with $T_{\mathrm{e}} \sim 2900 \pm 300 \mathrm{~K}$ (Luhman et al. 2003). To get a rough estimate of the stellar parameters, we assume that $B$ is coeval to the FUor, thus with an age between $5 \times 10^{5}$ and $10^{6}$ years (Kóspál et al. 2015). We adopt the evolutionary tracks from Baraffe et al. (2015), inferring $M_{*}=0.1 \pm_{0.08}^{0.1} M_{\odot}$ and $R_{*}=1.0 \pm_{0.6}^{0.7} R_{\odot}$.

We use the $J-K$ color excess of 2MASS J06593158$0405277 \mathrm{~B}$ and an $\mathrm{SpT}=\mathrm{M} 7$ (with $(J-K)_{0}=1$; see, e.g., Scholz et al. 2012) to estimate both upper and lower values of the visual extinction $\left(A_{\mathrm{V}}\right)$ toward the source, adopting the extinction law from Rieke \& Lebofsky (1985). Indeed, by assuming that the observed $E(J-K)$ is only due to the visual extinction, we get an upper limit of $A_{\mathrm{V}}=5 \mathrm{mag}$ from $A_{\mathrm{V}}=\left[(J-K)-(J-K)_{0}\right] / 0.17$. This is only an upper limit, because this source is young and thus part of its IR excess originates from veiling $\left(r_{\lambda}=F_{\mathrm{ex}, \lambda} / F_{*, \lambda}\right)$. The IR excess is thus

$$
E(J-K)=(J-K)-(J-K)_{0}+2.5 \log \left(\frac{1+r_{J}}{1+r_{K}}\right) .
$$

We cannot derive both $r_{J}$ and $r_{K}$ values. However, by assuming $r_{J}=0$ and fitting an SpT $=\mathrm{M} 7$ template, we then get $r_{K} \sim 2.6$, and we can infer a lower limit for the visual extinction of $A_{\mathrm{V}}=2.4 \mathrm{mag}$.

The spectrum of 2MASS J06593158-0405277 B (see upper panels of Figure 2) also displays $\mathrm{H}_{\mathrm{I}}$ lines in emission, namely the $\mathrm{Pa} \beta$ line at $1.282 \mu \mathrm{m}\left(F=(2.4 \pm 0.1) \times 10^{-15} \mathrm{erg} \mathrm{s}^{-1} \mathrm{~cm}^{-2}\right)$ and the $\mathrm{Br} \gamma$ line at $2.166 \mu \mathrm{m}\left(F=(6 \pm 2) \times 10^{-15} \mathrm{erg} \mathrm{s}^{-1} \mathrm{~cm}^{-2}\right)$. These lines are associated with ongoing accretion (see, e.g., Muzerolle et al. 1998; Natta et al. 2004; Caratti o Garatti et al. 2012), and can be employed to derive an estimate of the mass accretion rate (see, e.g., Natta et al. 2004, 2006). With the empirical relations by Muzerolle et al. (1998), this translates into an average accretion luminosity of $L_{\text {acc }}=0.005-0.01 L_{\odot}$. The mass accretion rate can then be expressed as $\dot{M}_{\text {acc }}=1.25 \times L_{\text {acc }} R_{*} / G M_{*} \quad$ (see, e.g., Caratti o Garatti et al. 2012). Inferred values are between $4 \times 10^{-9}$ and $8 \times 10^{-10}$ $M_{\odot} \mathrm{yr}^{-1}$, consistent with accretion rates found in young brown dwarfs (Natta et al. 2004; Mohanty et al. 2005; Joergens et al. 2013).

\subsection{The Disk, the Bump, and the FUor}

$J$ - and $K$-band continuum images in Figure 1 (left panels) display an elongated disk-like structure extending up to $\sim 200$ mas (or $\sim 90 \mathrm{AU}$ ) from the central source. Elliptical isophotes were fitted to the disk-like feature using the iraf STSDAS task isophote, starting from radii larger than the central PSF (i.e., $\sim 50$ mas and $\sim 70$ mas in the $J$ and $K$ band, respectively). The isophotal fit in the $J$ - band provides us with a semimajor axis P.A. of $52^{\circ} \pm 3^{\circ}$, and a ratio of the minor to major axes of $0.88 \pm 0.05$. Assuming circular symmetry, the system axis inclination with respect to the line of sight is $i=28^{\circ} \pm 6^{\circ}$. Results of the $K$-band analysis are less accurate but consistent with the previous ones, yielding $\mathrm{P}$. A. $=48^{\circ} \pm 10^{\circ}$ and $i=22^{\circ} \pm 12^{\circ}$. Our analysis thus indicates that this disk-like structure is almost face-on.

Another notable circumstellar feature around 2MASS J06593158-0405277 is the "bump" detected in continuum and line emission images (see Figure 1, central and right panels). This feature is not spatially resolved in the $J$ - and $K$-band images, but it is clearly detected after subtracting the rotated images (Figure 1, central panels) as an arc-shaped elongated feature, peaking at P.A. $\sim 233^{\circ}$ and $\sim 225^{\circ}$ in the $J$ 
and $K$ bands, respectively, and with $\mathrm{m}_{J} \sim 12.9 \mathrm{mag}$ and $\mathrm{m}_{K} \sim 10.6$ mag. In the continuum-subtracted $\mathrm{Pa} \beta$ and $\mathrm{Br} \gamma$ images (Figure 1, right panels), the "bump" emission appears as a point-like source, well fitted with a Gaussian profile. Its coordinates are R.A.(J2000) = 06:59:31.588, decl. $(\mathrm{J} 2000)=-04: 05: 27.86$, i.e., it is at $\Delta \alpha=-15$ mas, $\Delta \delta=-20$ mas from the FUor, that is, $\Delta r=25$ mas (or 11.25 AU), with a P.A. $=233$. 1 . A rough estimate of the $\mathrm{Pa} \beta$ and $\mathrm{Br} \gamma$ line fluxes gives $\sim 7 \times 10^{-15}$ and $2 \times 10^{-15} \mathrm{erg} \mathrm{s}^{-1} \mathrm{~cm}^{-2}$, respectively. The detection in both continuum-subtracted line images, the fact that these features are fitted with Gaussian profiles, and the presence of continuum emission in the PSFsubtracted $J$ - and $K$-band images strongly suggest that the "bump" is an additional closer young companion, which might have triggered the burst (see e.g., Reipurth \& Aspin 2004; Reipurth et al. 2014, p. 267). Moreover, in both $J$ and $K$ bands, the "bump" appears brighter than 2MASS J06593158$0405277 \mathrm{~B}$, hinting that, if it is a YSO, it might be more massive than the latter. However, as the "bump" is not spatially resolved in the continuum, other scenarios cannot be completely ruled out for the continuum emission, such as an uneven disk illumination (see, e.g., Dullemond \& Monnier 2010).

Finally, the lower panels of Figure 2 show the FUor flux calibrated spectrum $\left(m_{J}=8.63 \pm 0.05 \mathrm{mag}\right.$ and $m_{K}=7.45 \pm 0.04 \mathrm{mag}$ ), which only exhibits molecular and atomic features in absorption, as usually observed in FUor spectra, namely $\mathrm{H}_{2} \mathrm{O}, \mathrm{CO}, \mathrm{H}_{\mathrm{I}}(\mathrm{Pa} \beta$ and $\mathrm{Br} \gamma)$, Na I, Ca II, $\mathrm{K}$, $\mathrm{Mg}$ I, and Fe I. From these features we infer a SpT between K9 and M1, typical of FUor NIR spectra (see, e.g., Audard et al. 2014, p. 387). Notably, this spectrum closely resembles that of HBC 722, a well studied FUor (see Miller et al. 2011).

\section{DISCUSSION}

The detection of binaries or higher order systems in FUors is potentially of great importance, as interaction with a companion could be a triggering mechanism, although, in principle, there might be more than one. For example, a couple of FUors (V1057 Cyg and V1515 Cyg) are probably single systems (see Audard et al. 2014 and references therein), thus, in this case, internal instabilities seem to be the most straightforward process to consider. On the other hand, a large number of FUors and FUor candidates are binaries, and Bonnell \& Bastien (1992) first proposed that FUor events may be triggered by the passage of a close companion. Later on, Reipurth \& Aspin (2004) suggested that these objects might be newborn binaries, which arise from the breaking of unstable triple systems (see also Reipurth et al. 2014). As a consequence of the dynamical interaction, one object is ejected, typically the lowest mass component, whereas the remaining two objects spiral in toward each other perturbing their disks. The bursts would be then triggered by the close passage $(\sim 10 \mathrm{AU})$ of the nearest companion.

This scheme is a plausible explanation for what we are observing in 2MASS J06593158-0405277. The central object is a FUor, whose burst might have been triggered by a close companion (the "bump") located at $\sim 11 \mathrm{AU}$, and there is a more distant $(\sim 100 \mathrm{AU})$ and less massive object, which could be the third (ejected?) companion of a non-hierarchical system (e.g., Reipurth et al. 2014). Notably, the present configuration is precisely what would be expected from the dynamical evolution of a newborn triple system, where the least massive of three bodies is kept away from the two other bodies except for brief periastron passages, which eventually lead to the system dissolution (see, e.g., Reipurth \& Clarke 2001; Reipurth et al. 2010). At an age of $\sim 1 \mathrm{Myr}$ the observed system is statistically unlikely to be stable, and it will eventually eject the low-mass component after a periastron passage. This could be the first time that such scenario has been observed. Indeed companions in other FUors have been detected (e.g., FU Ori, Z CMa, L1551 IRS 5, RNO 1 B/C, AR 6 A/B; see, e.g., Reipurth \& Aspin 2004; Audard et al. 2014), but their relatively wide separation (from several tens up to hundreds of AU) suggests that these sources might be outlying companions.

In the case of 2MASS J06593158-0405277, the fact that both the "bump" and source "B" are young objects excludes the possibility that they might be background or foreground stars. Instead it is much more likely that they belong to the same star forming region, which is quite small and isolated, being composed of a few T Tauri stars (Kóspál et al. 2011). Their proximity to the FUor and the fact that the observed radial velocities of the HI lines are very similar in the three objects (average $v_{r} \sim 7 \pm 7 \mathrm{~km} \mathrm{~s}^{-1}$ with respect to the local standard of rest-LSR - and not corrected for the molecular cloud velocity) suggests that these sources might belong to the same system. Provided that the objects are physically linked, 2MASS J06593158-0405277 would then be one of the very few triple systems ever observed in FUors. That said, further high angular resolution observations are required to confirm the nature of the "bump" and the multiplicity of the 2MASS J06593158-0405277 system.

A.C.G., R.G.L., and T.P.R. were supported by Science Foundation Ireland, grant 13/ERC/I2907. S.K. acknowledges support from an STFC Ernest Rutherford Fellowship (ST/ J004030/1), Ernest Rutherford Grant (ST/K003445/1), and Marie Curie CIG grant (SH-06192).

Facilities: ESO/VLT.

\section{REFERENCES}

Audard, M., Ábrahám, P., Dunham, M. M., et al. 2014, Protostars and Planets VI, ed. H. Beuther et al. (Tucson, AZ: Univ. Arizona Press)

Baraffe, I., Homeier, D., Allard, F., \& Chabrier, G. 2015, A\&A, 577, A42 Baraffe, I., Vorobyov, E., \& Chabrier, G. 2012, ApJ, 756, 118

Beck, T. L., McGregor, P. J., Takami, M., \& Pyo, T.-S. 2008, ApJ, 676, 472 Bonnell, I., \& Bastien, P. 1992, ApJL, 401, L31

Caratti o Garatti, A., Garcia Lopez, R., Antoniucci, S., et al. 2012, A\&A, 538, A64

Clarke, C., Lodato, G., Melnikov, S. Y., \& Ibrahimov, M. A. 2005, MNRAS, 361,942

Cushing, M. C., Rayner, J. T., \& Vacca, W. D. 2005, ApJ, 623, 1115

Dullemond, C. P., \& Monnier, J. D. 2010, ARA\&A, 48, 205

Eisenhauer, F., Abuter, R., Bickert, K., et al. 2003, Proc. SPIE, 4841, 1548

Garcia Lopez, R., Caratti o Garatti, A., Weigelt, G., Nisini, B., \& Antoniucci, S. 2013, A\&A, 552, L2

Goodrich, R. W. 1987, PASP, 99, 116

Hackstein, M., Chini, R., Haas, M., Abraham, P., \& Kospal, A. 2014, ATel, 6838,1

Hartmann, L., \& Kenyon, S. J. 1996, ARA\&A, 34, 207

Herbig, G. H. 1977, ApJ, 217, 693

Hillenbrand, L. 2014, ATel, 6797, 1

Joergens, V., Herczeg, G., Liu, Y., et al. 2013, AN, 334, 159

Kim, B. G., Kawamura, A., Yonekura, Y., \& Fukui, Y. 2004, PASJ, 56, 313 Kóspál, Á, Ábrahám, P., Acosta-Pulido, J. A., et al. 2011, A\&A, 527, A133 Kóspál, Á, Ábrahám, P., Moór, A., et al. 2015, ApJL, 801, L5

Luhman, K. L., Stauffer, J. R., Muench, A. A., et al. 2003, ApJ, 593, 1093 Maehara, H., Kojima, T., \& Fujii, M. 2014, ATel, 6770, 1

Miller, A. A., Hillenbrand, L. A., Covey, K. R., et al. 2011, ApJ, 730, 80 
Modigliani, A., Hummel, W., Abuter, R., et al. 2007, arXiv:astro-ph/0701297 Mohanty, S., Jayawardhana, R., \& Basri, G. 2005, ApJ, 626, 498

Muzerolle, J., Calvet, N., \& Hartmann, L. 1998, ApJ, 492, 743

Natta, A., Testi, L., Muzerolle, J., et al. 2004, A\&A, 424, 603

Natta, A., Testi, L., \& Randich, S. 2006, A\&A, 452, 245

Rayner, J. T., Cushing, M. C., \& Vacca, W. D. 2009, ApJS, 185, 289

Reipurth, B., \& Aspin, C. 2004, ApJL, 608, L65

Reipurth, B., \& Clarke, C. 2001, AJ, 122, 432

Reipurth, B., Clarke, C. J., Boss, A. P., et al. 2014, Protostars and Planets VI, ed. H. Beuther (Tucson, AZ: Univ. Arizona Press)
Reipurth, B., Mikkola, S., Connelley, M., \& Valtonen, M. 2010, ApJL, 725, L56

Rieke, G. H., \& Lebofsky, M. J. 1985, ApJ, 288, 618

Scholz, A., Geers, V., Jayawardhana, R., et al. 2009, ApJ, 702, 805

Scholz, A., Muzic, K., Geers, V., et al. 2012, ApJ, 744, 6

Vorobyov, E. I., Baraffe, I., Harries, T., \& Chabrier, G. 2013, A\&A, 557, A35

Vorobyov, E. I., \& Basu, S. 2015, ApJ, in press (arXiv:1503.07888)

Zhu, Z., Hartmann, L., \& Gammie, C. 2010a, ApJ, 713, 1143

Zhu, Z., Hartmann, L., Gammie, C. F., et al. 2010b, ApJ, 713, 1134 